\title{
Implementation of external quality assessment of microscopy for improved parasite detection and confirmatory diagnosis of malaria in Tanzanian Military health facilities
}

Akili K. Kalinga ${ }^{1,2^{*}}$ (D, Saidi Mgata ${ }^{3}$, Reginald A. Kavishe2, Lucas Mahikwano ${ }^{3}$, Lucky Temu ${ }^{3}$, Christopher Mswanya ${ }^{4}$, Charles Mwanziva ${ }^{4}$, George Amoo ${ }^{5}$, Edwin Kamau ${ }^{6}$, Brian Vesely ${ }^{6}$ and Deus S. Ishengoma ${ }^{1,7,8}$

\begin{abstract}
Objective: Good quality microscopy is critical for accurate detection and confirmation of malaria parasite infections. Microscopy relies on the skills of technicians to prepare and read slides, high quality reagents, and a good program of internal and external quality control (EQA), which are lacking in most malaria endemic settings. This study was undertaken between January 2016 and December 2018 to pilot an EQA of microscopy for improved diagnosis of malaria and patient care in Tanzanian Military health facilities.

Results: Of all blood smears crosschecked $(n=4000)$ at baseline, only $38.5 \%$ were incorrectly diagnosed by laboratory staff with false positive and negative rates of $46.7 \%$ and $16.4 \%$, respectively. During the implementation of EQA, false positive and negative results decreased due to increased quality index of slide preparation and reading through supportive supervision, and retraining of laboratory personnel. There was a gradual increase of quarterly and annual total quality index for all laboratories, from 60\% in 2016 to $78 \%$ in 2017 and 90\% in 2018. The mean proficiency testing performance scores also increased from 75\% in 2016 to $82 \%$ in 2017 and to 90\% in 2018. Poor blood smear preparation and staining contributed to high false positive and negative rates while EQA helped in improvement of diagnostics.
\end{abstract}

Keywords: Malaria, Microscopy, External quality assessment, Proficiency testing, Total quality index, Military health facility, Tanzania

\section{Introduction}

Prompt parasitological detection and confirmation of parasite infections is recommended as an important pillar of malaria case management for improved care and treatment of febrile patients [1,2]. Despite recent introduction of rapid diagnostic tests, good quality microscopy is still considered a reference test for malaria

*Correspondence: kalingaaka@yahoo.com

${ }^{1}$ National Institute for Medical Research, Dar es Salaam, Tanzania

Full list of author information is available at the end of the article diagnosis [3, 4]. However, the effectiveness of malaria microscopy depends on maintaining a high level of competence and performance of laboratory staff, ensuring good-quality reagents, proper preparation and staining of blood smears and regular internal and external quality assessment, which are currently lacking in most malaria endemic countries $[4,5]$.

The World Health Organization (WHO) recommends for malaria endemic countries to implement a comprehensive external quality assessment (EQA) to ensure the quality of malaria diagnosis by microscopy $[4,6]$. The EQA

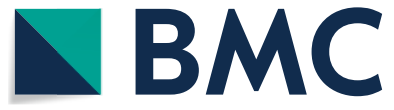

(c) The Author(s) 2020. This article is licensed under a Creative Commons Attribution 4.0 International License, which permits use, sharing, adaptation, distribution and reproduction in any medium or format, as long as you give appropriate credit to the original author(s) and the source, provide a link to the Creative Commons licence, and indicate if changes were made. The images or other third party material in this article are included in the article's Creative Commons licence, unless indicated otherwise in a credit line to the material. If material is not included in the article's Creative Commons licence and your intended use is not permitted by statutory regulation or exceeds the permitted use, you will need to obtain permission directly from the copyright holder. To view a copy of this licence, visit http://creativeco mmons.org/licenses/by/4.0/. The Creative Commons Public Domain Dedication waiver (http://creativecommons.org/publicdomain/ zero/1.0/) applies to the data made available in this article, unless otherwise stated in a credit line to the data. 
describes a method that allows testing conducted by a laboratory, testing site or individual user to be compared to that of a source outside the laboratory [7]. Traditionally, EQA programs focus exclusively on reading accuracy. However, the accuracy of microscopy is also critically dependent on the quality of blood smear preparation and staining procedures because poorly prepared and/or stained blood films directly reduces reading accuracy regardless of reading skill. Therefore, EQA through randomized slide re-checking of samples, and proficiency testing of laboratory staff are important malaria microscopy quality improvement program's (QIPs) that contribute to improvement of diagnosis, and ultimately the quality of patient care $[8,9]$.

Cross-checking is an important component of effective EQA that indicates whether a laboratory is providing accurate results, and can detect major deficiencies in laboratory performance due to low competence of staff, poor equipment, poor reagents, poor infrastructure or poor work practices [4]. Cross-checking malaria slides for quality of smear prepared by facility microscopists and readers' results is a standard EQA process recommended by the WHO for quality improvement [7]. Proficiency testing is another important aspect of EQA, which is implemented when a reference laboratory sends stained blood smear samples with known parasitaemias to testing laboratories for assessing and reading to determine the accuracy of laboratory staff. Upon reading and submission of the results, the reference laboratory gives feedback about the correct and incorrect reading results by individual laboratory staff, and overall laboratory performance. When under performance of routine malaria microscopy slide preparation and reading accuracy at health facilities is observed, that calls for interventions to improve the quality and performance of the laboratory procedures and training [9, 10]. Such interventions might include supportive supervision coupled with onsite training by supervisors from higher level laboratory $[6,11]$.

In Tanzanian military health facilities (MHFs), microscopy is commonly used due to shortage of rapid diagnostic tests (RDTs) as a result of unsustainable costs to meet the high demand of large influx of military recruits at the camps. Unfortunately, due to lack of expertise and financial resources, there is no microscopy EQA scheme. In this study, through Walter Reed Army Institute of Research of US, we piloted an EQA program in MHFs of Tanzania Peoples Defense Forces (TPDF) to provide objective data on the quality of malaria diagnosis.

\section{Main text}

\section{Materials and methods}

\section{Study area and populations}

The study was done in eight MHFs located in six malaria endemic regions across Tanzania. The MHFs included
Bulombora, Chita, Kaboya, Maramba, Mgambo, Msange, Ruvu and Rwamkoma.

The study population included patients admitted (inpatients) and not admitted (outpatients) at MHFs.

\section{Study design and period}

A cross-sectional study was conducted in selected facilities from January 2016 to December 2018.

\section{Sample size and sampling}

A total of 16 professional laboratory staff were purposively recruited in QIPs at their respective MHFs. A total of 16,000 slides with thick blood smears from all the laboratories at the MHFs formed a sampling frame. Of these, $20 \%(n=3200)$ were positive of which $65 \%(n=2080)$ were found and collected for assessment. A total of 1920 negative slides were also collected by selecting 10 slides each month using a random sampling method from each MHF in 10 rounds done on quarterly visits. For proficient testing, 2240 slides with standardized expert validated blood smears were available.

\section{Training of microscopists}

Prior to commencement of the microscopy QIPs at their MHFs and after competency assessment test, 16 microscopists trained as laboratory technicians and assistants who attended standard 2 weeks' microscopy course at Malaria Diagnostic Centre as per WHO curriculum participated in the study. The microscopists were assessed by three expert microscopists who were taking part in annual proficiency testing.

\section{Supply of laboratory equipment, reagents and consumables} Unlike before implementation of QIP, during quarterly site visits when EQA started, as part of the quality improvement program, MHFs were supplied sustainably with high quality malaria diagnostic equipments (microscopes, slide warmers, etc.), reagents (stains and fixatives) and laboratory consumables (slide preparation template, distilled water etc.).

\section{Quality control}

The smears were read for the first time by microscopists at MHFs. Two experienced expert microscopists performed the second reading as another microscopist performed the third read to break-tie the discordant results of the two assessors.

\section{Data collection}

Slide crosschecking Each quarter, assessors collected from storage boxes, slides that were 2-3 months old. Assessors selected in situ from the laboratory register, all reported positive and 10 negative blood slides each 
month. Negative slides were selected for re-reading at the reference laboratory, using a representative sampling protocol (Additional file 1) to ensure random distribution from the start, middle and end of working day and from variable days distributed across the month. Thick blood smears were assessed for quality of preparation, staining and reading accuracy. Slides were assessed macroscopically and microscopically on 12 key parameters namely, labelling, not cracked, not fixed, no fungus, no bacteria, no wash, size of smear, thickness of smear, uniformity of smear, correct staining and reading the results (Additional file 2). The assessment aimed at scoring total quality index (TQI) of laboratory as quality performance. The TQI is a combined score (\%) from all microscopists for the macroscopic and microscopic 12 parameters of blood smears at MHFs. It is the average score of all microscopists for a month, quarter and year under review.

Proficiency testing It was done using malaria slides provided by National Malaria Slide Bank (NMSB). The slides had been read and validated by four blinded National and International experts with WHO certification. During supervision visits, each laboratory staff was assigned to read 20 slides with known parasitaemia of which nine were negatives and 11 were positives with low $(<100$ asexual parasites/ $\mu \mathrm{l})$ or high $(\geq 100$ asexual parasites/ $\mu \mathrm{l})$ parasitaemia. The results for each laboratory staff was compared against known results of the samples then aggregated quarterly for each MHF. The quarterly average performance of proficiency testing was calculated for each participating MHF.

\section{Data analysis}

The data was managed using Microsoft Excel software and analyzed using STATA software (STATA Inc, TX, USA). Statistical significance was measured at $\mathrm{P}$-value $<0.05$. The results of the EQA was reported for 12 parameters using quality index indicators of well performed (over 75\%) for more than 9 parameters, fairly performed (50 to 75\%) between 6 and 9 parameters, and poorly performed $(<50 \%)$ for less than 6 parameters.

\section{Results}

\section{Characteristics of items for external quality assessment}

Of the 16 laboratory staff assessed, 13 were assistant health laboratory technologists and two were health laboratory technologists. A total of 4000 slides (1920 negative and 2080 positive) were selected for cross-checking while 2240 validated slides were given to laboratory staff for proficiency testing. Proficiency testing was done three quarters later compared to crosschecking that started earlier in quarter one of 2016.

\section{Slide crosschecking}

At the start of the EQA, the baseline data showed that preparation and staining of blood smears were well performed $(>75 \%)$ for more than half of parameters assessed. About one quarter of all parameters were performed fairly (between 50 and 75\%) while two parameters were performed poorly $(<50 \%)$. The smears read by facility staff, $61.5 \%$ were correctly diagnosed and $38.5 \%$ were incorrectly diagnosed (Fig. 1). For the smears reported as positive, $53.3 \%$ were true positive while $46.7 \%$ were false positive. With respect to smears reported as negative, $83.6 \%$ were true negative while only $16.4 \%$ were false negative. The rate of false positive over time decreased with an increase in the TQI of slide preparation; likewise, false negatives increased with a decrease in quality of preparation (Additional files 3 and 4).

Slide cross-checking revealed gradual increase of TQI from $52 \%$ in quarter one to $58 \%, 59 \%$ and $72 \%$ in quarters two, three and four respectively. Annual mean combined TQI for all studied laboratories increased from $60 \%$ in the first year (2016) to 78\% in the second year (2017) and 90\% in the third year (2018) (Fig. 2).

\section{Proficiency testing}

After 1 year, the average of proficiency test scores increased from $75 \%$ in quarter four in 2006 to $82 \%$ in quarter four in 2017. Generally, the mean proficiency testing performance scores showed an increasing trend from $75 \%$ in the first (2016) to $82 \%$ in the second (2017) and to $90 \%$ in the third year (2018) (Fig. 3).

\section{Discussion}

The findings from ten quarterly visits to MHFs demonstrated that supportive supervision contributed to identifying gaps, and through on-site training, a remarkable improvement in the quality of malaria diagnosis by microscopy was achieved. The increased performance was noticed among individual microscopists and the average aggregated results for each of the MHF as described previously $[12,13]$. In our view, the reported improvement could also be attributed to provision, and sustaining availability of quality laboratory equipment, reagents and consumables [12]. The observed improvements were apparent in the second quarter after implementing the QIPs during the first quarter, and was maintained in the subsequent quarters and over the three years.

Despite the observed increasing trend of overall laboratory performance for TQI (slide crosschecking) and combined scores (proficiency testing), few MHFs such as Ruvu did not show steady quarterly increasing trends. The reason behind this is the average performance in the 

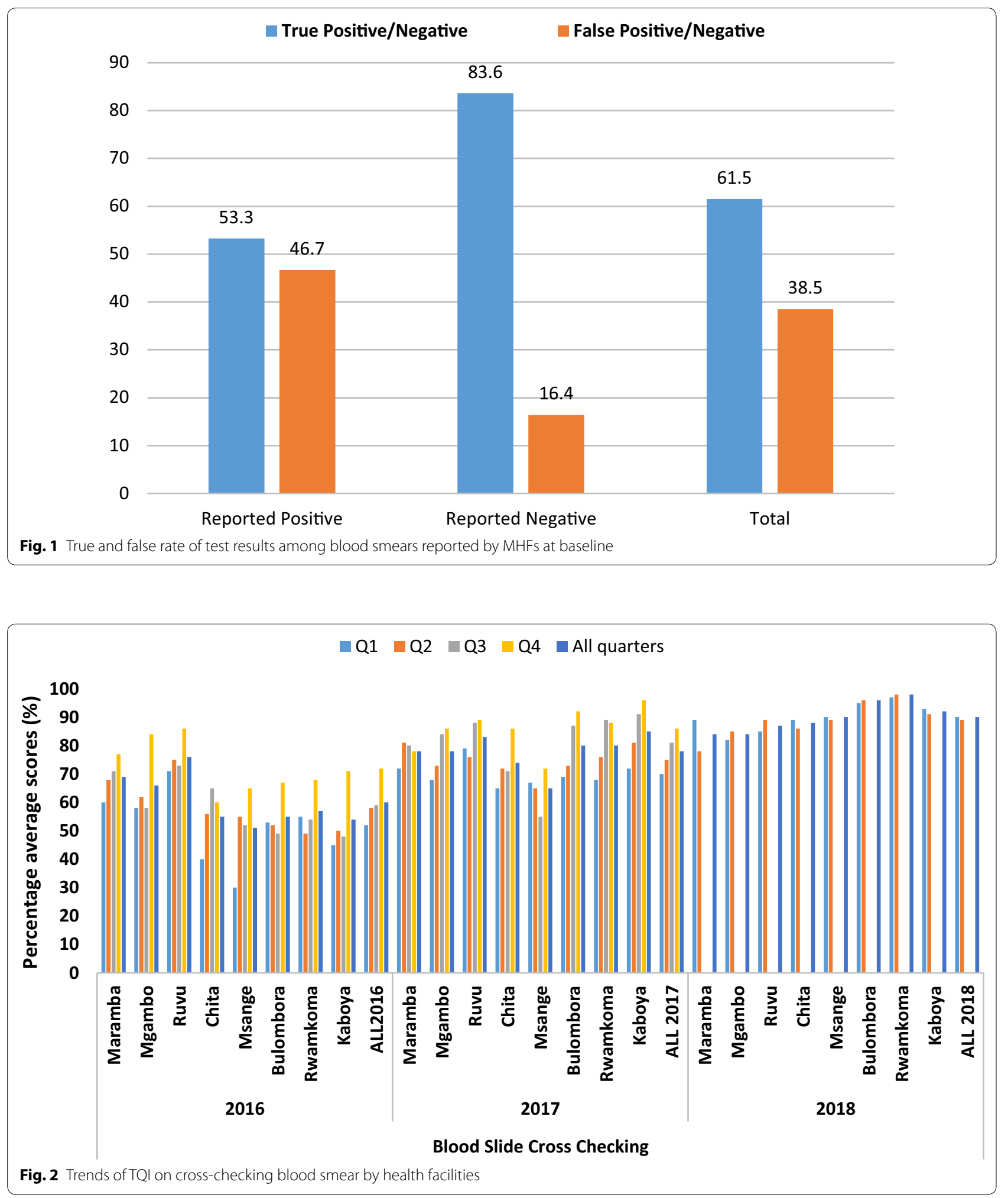

final score of the facility being pulled down by low performers among introduced new laboratory staff with less experience in microscopy in the reporting quarters compared to the previous ones when only trained experienced staffs performed well. This was confirmed when newly introduced laboratory staff were re-trained and 


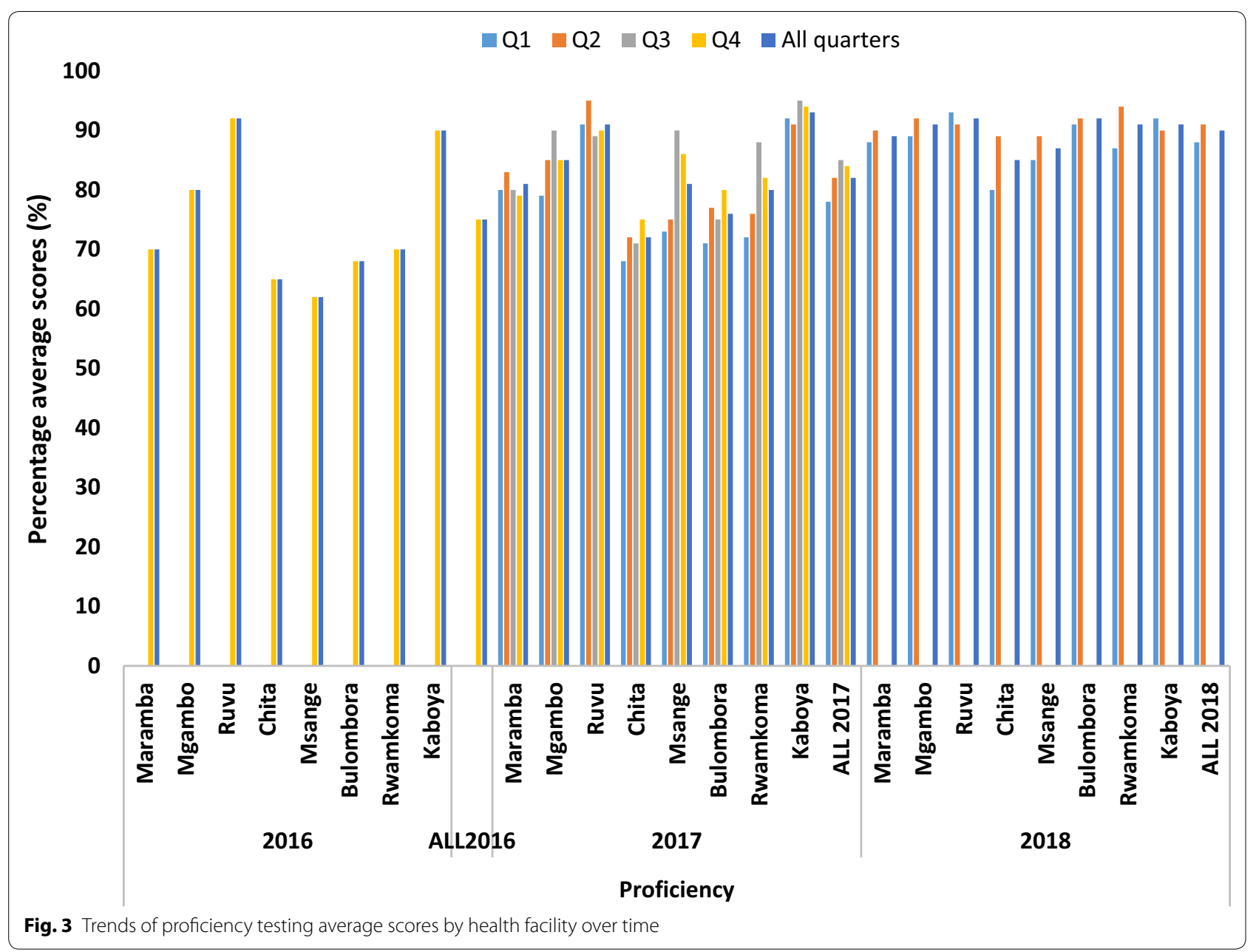

started to improve their individual scores, which ultimately raised the overall performance of the respective facility [14].

During the first quarter of assessment, the baseline data of combined TQI (from Crosschecking) was found to be $52 \%$ lower by $23 \%$ compared to $75 \%$ of a combined score (for proficiency testing) in the first quarter as well for all MHF. Similarly, the TQI in the first year (2016) was lower by $15 \%$ compared to combined proficiency testing scores for all MHFs. The possible explanation for the difference could be due to the effects of on-site retraining of laboratory staff that was conducted after the baseline assessment in the first quarter and before initiation of proficiency testing which started later in the fourth quarter of 2016. That is, by the time laboratory staff were assigned validated slides for proficiency testing, they had acquired reading skills during the initial training and retraining in the previous three quarterly visits.

Generally, a lower (52\%) mean preparation TQI for blood smear and high false positive rate (46.7\%) at the baseline, suggested that detection of malaria parasites in a poorly prepared and stained smear becomes more difficult and significantly reduces the sensitivity of microscopy. This was verified by re-training laboratory staff and improvements made thereafter. Unlike before implementation of EQA when the false test results were high, we observed improvement in total quality index that was associated with decrease of false positivity rates (graph S3) and vice versa. Likewise, false negatives increased with a decrease in quality of preparation (graph S4). That is, slides which had high quality with respect to preparation with contributed to increased accuracy in detection of the parasite and vice versa. Therefore, the results strongly suggest that poor preparation and staining of blood smear contribute significantly to the high rates of false results. Therefore, EQA programs should address quality of preparations and staining rather than focusing only on microscopists reading skills to ensure high quality of malaria diagnosis and case management. Thus, the overall improvement in the diagnostic services observed in this study 
was potentially attributed to increased site assessment scores as a result of implemented EQA at MHFs.

\section{Study limitations}

The implementation of supportive supervision was observational in nature making it difficult to isolate the effects of supportive supervision among other parameters in the observed improvement in quality diagnosis.

\section{Supplementary information}

Supplementary information accompanies this paper at https://doi. org/10.1186/s13104-020-05290-0.

\section{Additional file 1: Figure S1. Malaria microscopy external quality control} protocol.

Additional file 2: Figure S2. Preparation and staining parameters of blood smears on the slide.

Additional file 3: Figure S3. A graph showing trend of false positives versus preparation quality.

Additional file 4: Figure S4. A graph showing trend of false negatives versus preparation quality.

\section{Abbreviations}

EQA: External Quality Assessment; MHFs: Military Health Facilities; TPDF: Tanzania Peoples Defense Forces; TQI: Total Quality Index; QIPs: Quality Improvement Programmes; WHO: World Health Organization.

\section{Acknowledgements}

The authors are grateful to Walter Reed Army Institute of Research (WRAIR) in US for funding the main project in which these operational sub studies were nested. We are thankful to the Commission for Science and Technology $(\mathrm{COSTECH})$ in Tanzania and Fio Corporation in Canada for providing financial support for the PhD programme of Mr. Akili K. Kalinga, the corresponding author of this article. Authors are indebted to Tanzania Peoples Defense Forces (TPDF) and the National institute for Medical Research (NIMR) in Tanzania, and Henry Jackson Foundation Medical Research Institute (HJFMRI) in US for administrative support in the conduct of the studies. We owe gratitude to the Kilimanjaro Christian Medical University College (KCMUCo) in Tanzania for hosting and supervising Mr. Kalinga and enabling him accomplish his research milestones. We extend our sincere acknowledgements to all laboratory staff at MHFs who were involved in this study.

\section{Disclaimer}

Material has been reviewed by the Walter Reed Army Institute of Research. There is no objection to its presentation and/or publication. The opinions or assertions contained herein are the private views of the author, and are not to be construed as official, or as reflecting true views of the Department of the Army or the Department of Defense. The investigators have adhered to the policies for protection of human subjects as prescribed in AR 70-25.

\section{Authors' contributions}

SM \& AKK designed the study; AKK, SM, LM, CM, RAK and DSI drafted the manuscript. AKK, DSI, \& SM analyzed data. AKK, DSI, RAK, LT, CM, BV, EK, and SM revised the manuscript. EK, RK and BV reviewed and edited the manuscript. All authors read and approved the final manuscript.

\section{Funding}

Walter Reed Army Institute of Research (WRAIR) funded the main study on malaria surveillance and diagnostic quality improvement programme in Tanzania in which this survey was embedded. The Commission for Science and Technology (COSTECH) in Tanzania and Fio Corporation in Canada also provided funding for Mr. Akili K. Kalinga, the corresponding author of this article for pursuing a PhD programme at KCMCo in Tanzania. None of the funders had any role in the design of the study, data collection, analysis, interpretation of data and in writing the manuscript.

\section{Availability of data and materials}

The datasets used for this study are available on reasonable request from the corresponding author

\section{Ethics approval and consent to participate}

This study received approval from the Medical Research Coordinating Committee (MRCC) of the Tanzanian National Institute for Medical Research (certificate \# NIMR/HQs/R.8a/Nol. IX/1338) and the Ethics Review Committees of Kilimanjaro Christian Medical University College (KCMUCo) in Moshi, Tanzania (CRERC \# 818). It was also approved by the Walter Reed Army Institute of Research (WRAIR) in the US. The Tanzania Peoples Defense Forces (TPDF) headquarters and individual study camps granted permission prior to study commencement. Participants were recruited on voluntary bases after signing an informed consent.

\section{Consent for publication}

Not applicable.

\section{Competing interests}

Authors declare no competing interests.

\section{Author details}

${ }^{1}$ National Institute for Medical Research, Dar es Salaam, Tanzania. ${ }^{2}$ Kilimanjaro Christian Medical University College, Moshi, Tanzania. ${ }^{3}$ Henry Jackson Foundation Medical Research International, Dar es Salaam, Tanzania. ${ }^{4}$ Tanzania Peoples Defense Forces, Dar es Salaam, Tanzania. ${ }^{5}$ Forgyn Health Systems Consultants, Washington, DC, USA. ${ }^{6}$ Walter Reed Army Institute of Research, Washington, DC, USA. ${ }^{7}$ Faculty of Pharmaceutical Sciences, Monash University, Melbourne, Australia. ${ }^{8}$ Harvard T.H Chan School of Public Health, Harvard University, Boston, MA, USA.

Received: 2 April 2020 Accepted: 12 September 2020

Published online: 18 September 2020

\section{References}

1. WHO. Guidelines for theTreatment of Malaria. 3rd ed. Geneva: World Health Organization; 2015.

2. Gething PW, Battle KE, Bhatt S, Smith DL, Eisele TP, Cibulskis RE, et al. Declining malaria in Africa: improving the measurement of progress. Malar J [Internet]. 2014;13(1):1-5.

3. WHO/FIND/CDC. Malaria Rapid Diagnostic Test Per formance: Summar y results of WHO product testing of malaria RDTs: rounds 1-6 (2008-2015) [Internet]. Vol. 6. 2015

4. WHO/CDC/CLSI. Malaria Microscopy quality assurance mannual; Version 2. ; ISBN 978924154939 4. 2016. 113-124.

5. Laktabai J, Platt A, Menya D, Turner EL, Aswa D, Kinoti S, et al. A mobile health technology platform for quality assurance and quality improvement of malaria diagnosis by community health workers. PLOS ONE. 2018;13(2):1-14.

6. Yitbarek T, Nega D, Tasew G, Taye B, Desta K. Performance evaluation of malaria microscopists at defense health facilities in Addis Ababa and its surrounding areas, Ethiopia. PLOS ONE. 2016:11(11):1-11.

7. WHO/CDC/CLSI. Overview of External Quality Assessment (EQA):module 10, content sheet 10-1. Geneva: WHO; 2016.

8. Simundic A-M, Topic E, Nikolac N, Lippi G. Special Quality in laboratory diagnostics: from theory to practice Izvorni issue: znanstveni članak. Biochem Medica. 2010:8:154-9.

9. James D, Ames D, Lopez B, Still R, Simpson W, Twomey P. External quality assessment: best practice. J Clin Pathol Eng. 2014:67:651-5.

10. Carter JY. Special issue: external Quality Assessment in Laboratory Medicine Original papers External quality assessment in resource-limited countries. Biochemia medica. 2017;27:97-109.

11. Mayengue PI, Kouhounina Batsimba D, Dossou-Yovo LR, Niama RF, Macosso L, Pembet Singana B, et al. Evaluation of Routine Microscopy Performance for Malaria Diagnosis at Three Different Health Centers in Brazzaville, Republic of Congo. Malar Res Treat. 2018;2018:1-6. 
12. Luckett R, Mugizi R, Lopes S, Etossi RC, Allan R. The role of laboratory supervision in improving the quality of malaria diagnosis: a pilot study in Huambo, Angola. Am J Trop Med Hyg. 2016;94(3):659-62.

13. Worges M, Whitehurst N, Yamo E, Moonga H, Yukich J, Benavente L. Outreach training and supportive supervision for malaria case management in Zambia: the effects of focused capacity building on indicators of diagnostic and clinical performance. Malar J. 2018;17(1):1-16.

14. Kalinga A, Ishengoma D, Kavishe R, Temu L, Mswanya C, Mwanziva C, et al. The use of Fionet technology for external quality control of malaria rapid diagnostic tests and monitoring health workers' performance in rural military health facilities in Tanzania. PLoS ONE. 2018;13(12):1-18.

\section{Publisher's Note}

Springer Nature remains neutral with regard to jurisdictional claims in published maps and institutional affiliations.
Ready to submit your research? Choose BMC and benefit from:

- fast, convenient online submission

- thorough peer review by experienced researchers in your field

- rapid publication on acceptance

- support for research data, including large and complex data types

- gold Open Access which fosters wider collaboration and increased citations

- maximum visibility for your research: over $100 \mathrm{M}$ website views per year

At BMC, research is always in progress.

Learn more biomedcentral.com/submissions 